\title{
Methodological quality of systematic reviews on treatments for depression: a cross-sectional study
}

\author{
V. C. H. Chung ${ }^{1,2}$, X. Y. Wu ${ }^{2,3 *}$, Y. Feng ${ }^{1}$ t, R. S. T. Ho ${ }^{1}$ t, S. Y. S. Wong ${ }^{1}$ and D. Threapleton ${ }^{1}$ \\ ${ }^{1}$ Jockey Club School of Public Health and Primary Care, The Chinese University of Hong Kong, Hong Kong \\ ${ }^{2}$ Cochrane Hong Kong, The Chinese University of Hong Kong, Hong Kong \\ ${ }^{3}$ Hong Kong Institute of Integrative Medicine, The Chinese University of Hong Kong, Hong Kong
}

\begin{abstract}
Aims. Depression is one of the most common mental disorders and identifying effective treatment strategies is crucial for the control of depression. Well-conducted systematic reviews (SRs) and meta-analyses can provide the best evidence for supporting treatment decision-making. Nevertheless, the trustworthiness of conclusions can be limited by lack of methodological rigour. This study aims to assess the methodological quality of a representative sample of SRs on depression treatments.
\end{abstract}

Methods. A cross-sectional study on the bibliographical and methodological characteristics of SRs published on depression treatments trials was conducted. Two electronic databases (the Cochrane Database of Systematic Reviews and the Database of Abstracts of Reviews of Effects) were searched for potential SRs. SRs with at least one meta-analysis on the effects of depression treatments were considered eligible. The methodological quality of included SRs was assessed using the validated AMSTAR (Assessing the Methodological Quality of Systematic Reviews) tool. The associations between bibliographical characteristics and scoring on AMSTAR items were analysed using logistic regression analysis.

Results. A total of 358 SRs were included and appraised. Over half of included SRs $(n=195)$ focused on non-pharmacological treatments and harms were reported in $45.5 \%(n=163)$ of all studies. Studies varied in methods and reporting practices: only $112(31.3 \%)$ took the risk of bias among primary studies into account when formulating conclusions; 245 $(68.4 \%)$ did not fully declare conflict of interests; 93 (26.0\%) reported an 'a priori' design and 104 (29.1\%) provided lists of both included and excluded studies. Results from regression analyses showed: more recent publications were more likely to report 'a priori' designs [adjusted odds ratio (AOR) 1.31, 95\% confidence interval (CI) 1.09-1.57], to describe study characteristics fully (AOR 1.16, 95\% CI 1.06-1.28), and to assess presence of publication bias (AOR 1.13, 95\% CI 1.06-1.19), but were less likely to list both included and excluded studies (AOR 0.86, 95\% CI 0.81-0.92). SRs published in journals with higher impact factor (AOR 1.14, 95\% CI 1.04-1.25), completed by more review authors (AOR $1.12,95 \%$ CI 1.01-1.24) and SRs on non-pharmacological treatments (AOR 1.62, 95\% CI 1.01-2.59) were associated with better performance in publication bias assessment.

Conclusion. The methodological quality of included SRs is disappointing. Future SRs should strive to improve rigour by considering of risk of bias when formulating conclusions, reporting conflict of interests and authors should explicitly describe harms. SR authors should also use appropriate methods to combine the results, prevent language and publication biases, and ensure timely updates.

Received 29 July 2016; Accepted 3 April 2017; First published online 2 May 2017

Key words: Depression, evidence-based medicine, meta-analysis, randomised controlled trials as topic, review literature as topic.

\section{Introduction}

As one of the most common mental disorders, more than 350 million people suffer from depression globally (World Health Organization, 2012). Aside from being a leading cause of disability worldwide (World

\footnotetext{
† These authors contributed equally to this paper.

* Address for correspondence: X. Y. Wu, Room 509, JC School of Public Health Building, Prince of Wales Hospital, Shatin, NT, Hong Kong.

(Email: wuxinyin@cuhk.edu.hk)
}

Health Organization, 2012), depression is also a significant cause of mortality given its strong links with suicide (Mykletun et al. 2009). Because depression is often chronic, patients depend heavily on both pharmacological and non-pharmacological treatment strategies (Cipriani et al. 2011). There are numerous treatments available for depression and it is important for clinicians to make informed decisions on their use, based on the best available clinical evidence. As a major approach for synthesising results from clinical trials, systematic reviews (SRs) use explicit and reproducible 
methods to identify, critically appraise and summarise all clinical trial results that meet pre-defined eligibility criteria (Higgins \& Green, 2011). It is a well-established method in evidence-based healthcare, distinguishing itself from narrative reviews which are prone to bias due to the lack of transparency (Higgins \& Green, 2011). Within a SR, meta-analysis is a common statistical method that allows quantitative synthesis of trial results, allowing estimation of the treatment effect with higher precision and permitting exploration of heterogeneity in patient outcomes (Higgins \& Green, 2011). A methodologically rigourous and up-to-date SR can provide the best clinical evidence for supporting treatment decision-making (Mulrow et al. 1997).

Despite high publication volume and wide acceptance among healthcare professionals (Moher et al. 2007), SRs should not be regarded as the 'best' sources of evidence without critical appraisal of included studies. A SR with methodological deficiencies might overestimate or underestimate treatment effect, draw biased conclusions and subsequently mislead decisionmaking (Mulrow et al. 1997). Evidence-users, including clinicians, policy-makers, managers and patients are advised not to accept the results and conclusions from SRs without critical appraisal of included evidence (Fleming et al. 2014). At present the quality of summary evidence to guide decision-makers in this area is unknown as no previous work has comprehensively examined the methodological quality of SRs focused on treatment for depression. This work will inform the state-of-science in the field and provide insight on how methodological quality may be improved in the future.

In this cross-sectional study, we aim to: (1) describe the bibliographical characteristics of a representative sample of SRs on depression treatments; (2) appraise the methodological quality of a representative sample of SRs on depression treatments using the AMSTAR (Assessing the Methodological Quality of Systematic Reviews) tool (Shea et al. 2007); and (3) examine the relationship between methodological rigour and bibliographical features.

\section{Materials and methods}

\section{Eligibility criteria}

All SRs that performed at least one meta-analysis of any depression treatment were eligible, without restrictions on publication year. We only included SRs with at least one meta-analysis in order to ensure that all 11 AMSTAR items were applicable during the critical appraisal process (Burda et al. 2016). SRs must have pooled primary studies recruiting patients with depression who were diagnosed using an established criteria or questionnaire [e.g., the Diagnostic and Statistical Manual of Mental Disorders, Third edition (American Psychiatric Association, 1980) or the Hamilton Rating Scale for Depression (Hamilton, 1960)]. Narrative reviews, protocols, network meta-analyses, SRs without meta-analysis or SRs focused on the diagnosis of depression were excluded. The most up-to-date version was selected when a SR had been updated.

\section{Literature search}

We applied the keyword 'depress"' in the Cochrane Database of Systematic Reviews (CDSR) and the Database of Abstracts of Reviews of Effects (DARE), of which the former contains all Cochrane reviews and the later indexes non-Cochrane SRs (Cochrane Community (beta) 2015). Databases were searched from inception until February 2017, with no language restriction.

\section{Literature screening, selection and data extraction}

The title and abstract of retrieved citations was screened independently by two authors (Feng and Ho). Full texts of potentially eligible citations were then retrieved, and eligibility was further assessed by these two authors, independently. Data extraction was again performed independently by two reviewers (Feng and Ho), with discrepancy resolved by referring to the original publications. Persistent disagreements were resolved by consensus adjudication led by a third author (Chung).

\section{Methodological quality assessment}

The methodological quality of included SRs was assessed with the AMSTAR (Shea et al. 2007). The 11 item AMSTAR is an established instrument for appraising the methodological quality of SRs (Shea et al. 2007). AMSTAR is a well-evaluated assessment tool with good agreement, construct validity and feasibility, as demonstrated in validation studies (Shea et al. 2009). It has been applied to evaluate the methodological quality of SRs in many clinical areas, including diabetes mellitus (Wu et al. 2016b), hypertension (Wu et al. 2016a), Chinese herbal medicine (Chung et al. 2015), chronic obstructive pulmonary disease (Ho et al. 2015), influenza vaccination (Remschmidt et al. 2014), orthodontics (Papageorgiou et al. 2011) and oral healthcare interventions (Sequeira-Byron et al. 2011). Performance of the included SRs on each of the 11 items is reported separately, with no calculation of the overall score, as there is no empirical evidence on how to assign an appropriate weighting for each item (Shea et al. 2009). The detailed assessment form 
for AMSTAR is presented in online Appendix A. Judgements were assigned for each SR according to standard guidelines and each item was classed as 'yes', 'no' or 'cannot answer/not reported', as appropriate. The methodological quality was assessed independently by two assessors (Feng and Ho). In case of discrepancy, a third researcher (Chung) was consulted.

\section{Data analysis}

Bibliographical characteristics and the AMSTAR assessment results were analysed with descriptive statistics. Data were summarised as frequencies, percentages, medians and ranges. The associations between bibliographical characteristics and scoring on AMSTAR items were analysed using logistic regression analysis. Adjusted odds ratio (AOR) was calculated to measure the association between bibliographical characteristics and scoring on AMSTAR items. Seven potential predictors were examined for SR quality: Cochrane review $v$. non-Cochrane review, update review $v$. original review; year of publication (continuous variable); impact factor of the journal at the year before the SR published (continuous variable); number of authors (continuous variable); location of corresponding author Europe $v$. non-Europe and pharmacological $v$. nonpharmacological treatment. We used the 'Enter' method to include all seven predictors in the regression model. Predictors that showed statistical significance were reported in Table 3, results on remaining predictors will be available on request. A $p$-value $<0.05$ was considered as reaching statistical significance. The Hosmer-Lemshow (HL) test was applied to evaluate the degree of model-fitting for logistic regression, with a $p$-value $>0.1$ indicating good model-fitting. All the statistical analyses were conducted with the Statistical Package for Social Sciences (SPSS) 21.0.

\section{Results}

\section{Bibliographical characteristics of SRs}

We identified 1653 records through literature searches, of which 1196 were excluded as ineligible. The remaining 358 were included in this study and the literature selection flow can be found in Fig. 1. The 358 SRs included a total of 8034 primary studies and 877010 participants, and a full list of included SRs can be found in online Appendix B. The included SRs were published between 1995 and 2016, with a median publication year of 2011. Bibliographical characteristics of the included SRs are presented in Table 1. More than half $(54.5 \%)$ of the SRs focused on non-pharmacological treatments, the remaining 163 SRs (45.5\%) reporting on pharmacological treatments for depression. Of the pharmacological and non-pharmacological treatment SRs, 34.4 and $71.3 \%$ did not report treatment harms, respectively.

Sixty-two SRs $(17.3 \%)$ were Cochrane reviews and $56(15.6 \%)$ SRs were an update of a previous SR. Most SRs (72.6\%) were published in journals with impact factors lower than 5 and only 12 (3.4\%) were accepted by journals with impact factors of higher than 10 . The median review team size was 4 (range 1-13). In $46.9 \%$ of the SRs, the corresponding authors were based in Europe, and around one quarter $(22.3 \%)$ were from America. Funding source locations had a similar distribution to that of the corresponding authors, but such information was not reported in more than half of the SRs.

The majority of SRs (96.6\%) searched English databases (that also index non-English articles) and 53.1\% of SRs report including articles of any language. Only $58(16.2 \%)$ SRs conducted searches in non-English databases. English databases were defined as those operating in English but which may index articles with full-text articles not in English (such as DARE). Non-English databases include any that are not operated in English. Furthermore, 74 SRs (20.7\%) reviewed English publications exclusively. Most SRs (91.3\%) included randomised controlled trials (RCTs) alone, $18(5.0 \%)$ included RCTs and observational studies and only six (1.7\%) SRs included solely observational studies (Table 1).

\section{Methodological quality of SRs}

Results of the methodological quality assessment are reported according to each AMSTAR item in Table 2. The majority of SRs (95.8\%) described characteristics of included studies and conducted comprehensive literature searches (88.5\%). Although $75.7 \%$ of SRs assessed and documented the risk of bias in the primary studies, only $31.3 \%$ took the risk of primary study bias into account when drawing conclusions. Around one quarter $(25.7 \%$ ) of SRs used inappropriate methods to combine findings during meta-analyses. Furthermore, most SRs had poor performance in the following three methodological aspects: (i) declaring conflict of interests for all included primary studies and the SR itself (31.6\%); (ii) providing an 'a priori' published protocol $(26.0 \%)$; and (iii) providing lists for both included and excluded studies (29.1\%). Common shortcomings on reporting of SR methodology were: one third (33.2\%) of the SRs did not report sufficient information to determine whether duplicate study selection and data extraction were performed, and $20.4 \%$ did not clearly state whether they included grey literature or not. 


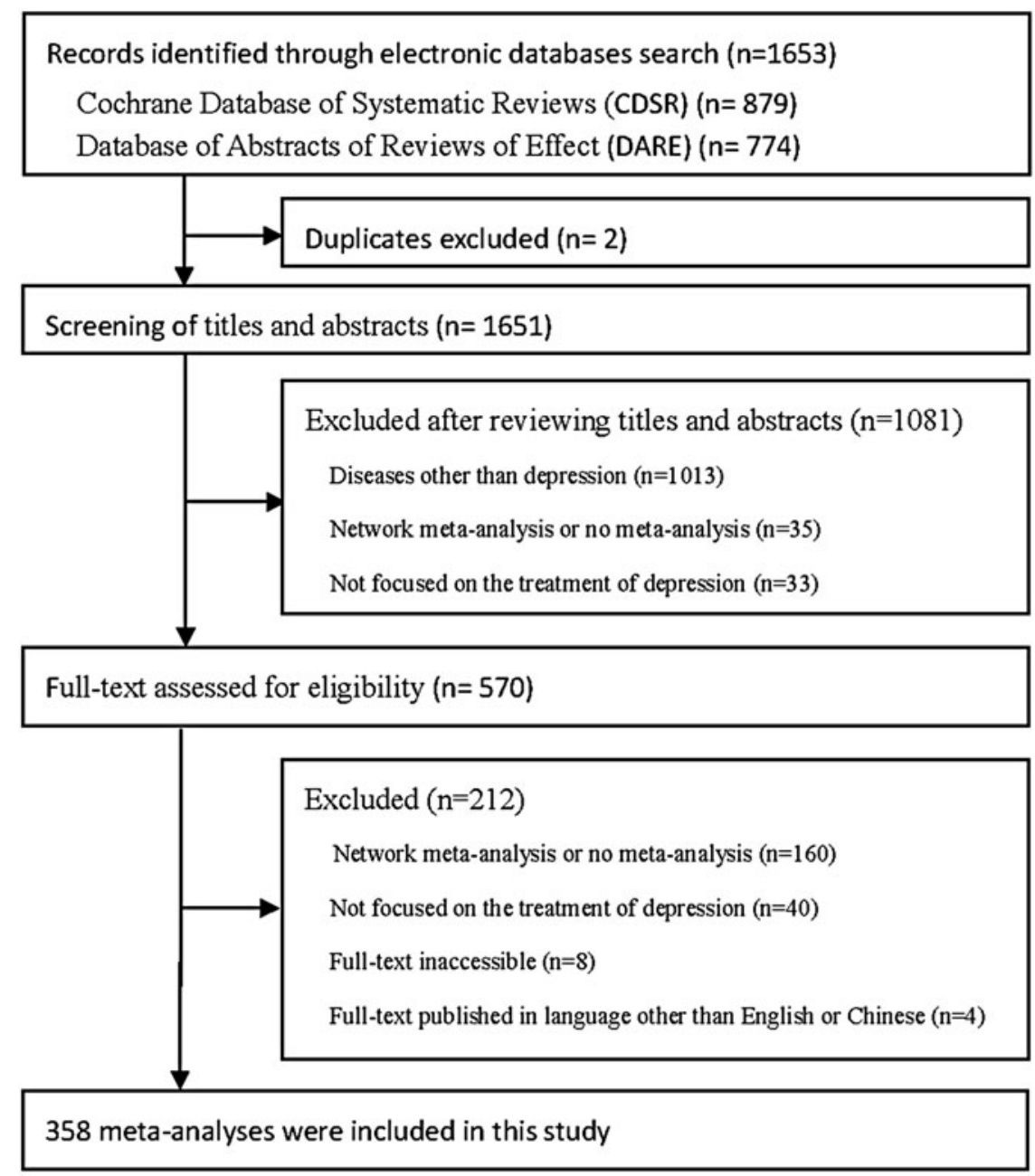

Fig. 1. Sampling of meta-analyses on depression treatments: flow chart.

\section{Predictors of methodological quality}

In logistic regression analyses, significant associations between bibliographical characteristics and methodological quality were observed in five AMSTAR items (Table 3), however the majority of characteristics were not associated with any other AMSTAR items. SRs published more recently were more likely to provide an 'a priori' design (AOR for each additional year: $1.31,95 \%$ confidence interval (CI) 1.09-1.57), to describe characteristics of the included studies (AOR 1.16, 95\% CI 1.06-1.28) and to assess publication bias (AOR 1.13, 95\% CI 1.06-1.19). However, they were less likely to list both included and excluded studies (AOR 0.86, 95\% CI 0.81-0.92). Three predictors were identified for better performance in publication bias assessment: higher impact factor (AOR per 1 impact factor unit increase: $1.14,95 \%$ CI 1.04-1.25), SRs completed by more review authors (AOR for each additional author: 1.12, 95\% CI 1.01-1.24) and SRs on non-pharmacological treatments (AOR 1.62, 95\% CI
1.01-2.59). SRs completed by teams with a European corresponding author (AOR 3.90, 95\% CI 1.70-8.97) were more likely to provide an 'a priori' design, and to combine the results using appropriate statistical methods (AOR 1.76, 95\% CI 1.05-2.93). Finally, SRs on non-pharmacological treatments were less likely to provide list of included and excluded studies (AOR $0.42,95 \%$ CI $0.20-0.86$ ). The $p$-values of all HL tests were $>0.1$, indicating good model fit for all logistic regression analyses.

\section{Discussion}

We have included a representative sample of SRs ( $n=$ 358) published between 1995 and 2016, which had synthesised evidence on the effectiveness of depression treatments. This reflects an acceptance of the evidencebased approach in making decisions on depression treatments. More than half of these SRs were conducted by teams led by European researchers. 
Table 1. Bibliographical characteristics of 358 included systematic reviews on depression treatments

\begin{tabular}{|c|c|}
\hline Bibliographical characteristics & Results* \\
\hline Cochrane review & $62(17.3)$ \\
\hline Non-Cochrane review & $296(82.7)$ \\
\hline An update of a previous review & $56(15.6)$ \\
\hline An update of a previous Cochrane review & $16(25.8)$ \\
\hline An update of a previous non-Cochrane review & $40(13.5)$ \\
\hline Publication year & 2011 (1995-2016) \\
\hline Publication journal impact factor & $3.1(0.0-21.5)$ \\
\hline Number of review authors & $4(1-13)$ \\
\hline SRs with only one author & $9(2.5)$ \\
\hline \multicolumn{2}{|l|}{ Location of corresponding author } \\
\hline Europe & $168(46.9)$ \\
\hline America & $80(22.3)$ \\
\hline Asia & $72(20.1)$ \\
\hline Oceania & $36(10.1)$ \\
\hline Africa & $1(0.3)$ \\
\hline Not reported & $1(0.3)$ \\
\hline \multicolumn{2}{|l|}{ Type of treatment } \\
\hline Non-pharmacological & $195(54.5)$ \\
\hline Pharmacological & $163(45.5)$ \\
\hline Primary studies in SR & $8034(22.4 \pm 29.8)$ \\
\hline Participants in included primary studies & $877010(2463.5 \pm 4001.2)$ \\
\hline SRs reporting intervention harms & $163(45.5)$ \\
\hline Non-pharmacological treatments & $56(28.7)$ \\
\hline Pharmacological treatments & $107(65.6)$ \\
\hline \multicolumn{2}{|l|}{ Funding location of the SR } \\
\hline Europe & $78(21.8)$ \\
\hline America & $53(14.8)$ \\
\hline Asia & $34(9.5)$ \\
\hline Oceania & $6(1.7)$ \\
\hline Not reported & $187(52.2)$ \\
\hline SRs that searched English databases & $346(96.6)$ \\
\hline SRs that searched non-English databases & $58(16.2)$ \\
\hline \multicolumn{2}{|l|}{ Report year span of search } \\
\hline Yes, reported both starting and ending years & $262(73.2)$ \\
\hline Partially, only reported starting years & $63(17.6)$ \\
\hline Not mentioned & $33(9.2)$ \\
\hline \multicolumn{2}{|l|}{ Search terms reported for one or more electronic databases } \\
\hline Topics/free text/keywords/MeSH & $284(79.3)$ \\
\hline Full Boolean & $40(11.2)$ \\
\hline Readers are referred elsewhere for full search strategy & $11(3.1)$ \\
\hline No research term & $23(6.4)$ \\
\hline \multicolumn{2}{|l|}{ Eligibility of study design } \\
\hline RCT only & $327(91.3)$ \\
\hline RCT and observational studies & $18(5.0)$ \\
\hline Observational studies only & $6(1.7)$ \\
\hline Not mentioned & $7(2.0)$ \\
\hline \multicolumn{2}{|l|}{ Eligibility criteria based on language of publication } \\
\hline English and non-English & $190(53.1)$ \\
\hline English publications only & $74(20.7)$ \\
\hline Not reported & $94(26.3)$ \\
\hline Included a PRISMA-like flow diagram & $208(58.1)$ \\
\hline
\end{tabular}

*Values are $n(\%)$, median (range) or $\mathrm{n}($ mean \pm S.D.).

RCT, randomised controlled trial; SR, systematic review; PRISMA, The Preferred Reporting Items for Systematic Reviews and Meta-analyses. 
Table 2. Methodological quality of 358 included systematic reviews on depression treatments

\begin{tabular}{|c|c|c|c|}
\hline Individual AMSTAR items & Yes $(\%)$ & No $(\%)$ & Can't answer (\%) \\
\hline 1. Was an 'a priori' design provided? & $93(26.0)$ & $265(74.0)$ & NA \\
\hline 2. Was there duplicate study selection and data extraction? & $165(46.1)$ & $74(20.7)$ & $119(33.2)$ \\
\hline 3. Was a comprehensive literature search performed? & $317(88.5)$ & $30(8.4)$ & $11(3.1)$ \\
\hline $\begin{array}{l}\text { 4. Was the status of publication used as an inclusion criterion } \\
\text { (i.e., grey literature would be included, if located)? }\end{array}$ & $191(53.3)$ & $94(26.3)$ & $73(20.4)$ \\
\hline 5. Was a list of studies (both included and excluded) provided? & $104(29.1)$ & $254(70.9)$ & NA \\
\hline 6. Were the characteristics of the included studies provided? & $343(95.8)$ & $15(4.2)$ & NA \\
\hline 7. Was the scientific quality of the included studies assessed and documented? & $271(75.7)$ & $87(24.3)$ & NA \\
\hline $\begin{array}{l}\text { 8. Was the scientific quality of the included studies used appropriately in } \\
\text { formulating conclusions? }\end{array}$ & $112(31.3)$ & $246(68.7)$ & NA \\
\hline 9. Were the methods used to combine the findings of studies appropriate? & $266(74.3)$ & $92(25.7)$ & NA \\
\hline 10. Was the likelihood of publication bias assessed? & $196(54.7)$ & $162(45.3)$ & NA \\
\hline $\begin{array}{l}\text { 11. Were the sources of support for both the systematic review and the included } \\
\text { primary studies reported? }\end{array}$ & $113(31.6)$ & $245(68.4)$ & NA \\
\hline
\end{tabular}

AMSTAR, Assessing the Methodological Quality of Systematic Reviews; NA, not applicable.

However, the methodological quality of these SRs is low, especially in the aspects of: (i) ensuring comprehensive literature search and being up to date; (ii) considering risk of bias when drawing conclusions and use of appropriate statistical approaches; (iii) declaring conflict of interests transparently; and (iv) the reporting of methodological details and treatment harms.

\section{Ensuring comprehensive literature search}

With regards to literature search, SR limitations may not be apparent at the first instance as $88.5 \%$ had conducted a 'comprehensive' search according to the AMSTAR standard. However, our data showed that only $16.2 \%$ performed searches in non-English databases. Although most of the included SRs searched two or more databases indexing non-English articles, we cannot exclude the possibility of language bias in study selection, as $20.7 \%$ included only English publications, and $26.3 \%$ did not describe eligibility criteria with regards to language. Empirical evidence has suggested that inclusion of only English publications in SRs can increase the risk of overestimation (Egger et al. 1997) or underestimation (Jüni et al. 2002) of treatment effects. Furthermore, the addition of non-English studies will improve generalisability and precision of pooled estimates (Morrison et al. 2012), as searches in non-English databases may allow identification of a large number of additional primary studies conducted in non-English-speaking populations (Wu et al. 2013; Cohen et al. 2015). Future SRs should set no eligibility criteria based on language, and English-speaking reviewers may reduce their burden by using recommended screening strategies (Busse et al. 2014).
Another methodological limitation is the possible exclusion of grey literature (Shea et al. 2007). Among included SRs, $26.3 \%$ did not include grey literature and $20.4 \%$ did not provide information on this issue. Current evidence suggested that exclusion of grey literature causes underrepresentation of negative results, and subsequently leads to overestimation of the treatment effects (Dwan et al. 2008). While SRs conducted by more authors, published more recently, published in higher impact factor journals or focused on nonpharmacological treatments are more likely to detect publication bias stemming from this problem, 45.3\% of the SRs did not assess publication bias and thus their reported effect size may be questionable. Aside from avoiding language and publication bias, trustworthiness of SR results also depends on whether the search is up-to-date (Moher \& Tsertsvadze, 2006).

\section{Considering risk of bias and use of appropriate statistics}

In SRs, assessing risk of bias of primary studies is of crucial importance as this will gauge the validity of meta-analytic results. Unfortunately, $24.3 \%$ of SRs did not appraise their included primary studies. Furthermore, only $31.3 \%$ explicitly consider risk of bias of primary studies when drawing conclusions. This implies that the 'garbage in, garbage out' (Egger et al. 2001) problem could be prevalent among SRs on depression treatments, echoing studies in other fields (Moja et al. 2005; Ho et al. 2015). Use of an inappropriate statistical method for pooling findings in $25.7 \%$ of the SRs represents another threat to validity. It is recommended that a multidisciplinary team 
Table 3. Association between characteristics of depression treatments systematic reviews and methodological quality: Logistic regression analysis

\begin{tabular}{|c|c|c|c|}
\hline AMSTAR item (dependent variable) & Predictors & Adjusted OR (95\% CI) & $p^{*}$ \\
\hline \multirow[t]{2}{*}{ 1. Was an 'a priori' design provided? $\dagger$} & Publication year & $1.31(1.09-1.57)$ & 0.004 \\
\hline & Corresponding author from Europe & $3.90(1.70-8.97)$ & 0.001 \\
\hline \multirow{2}{*}{$\begin{array}{l}\text { 5. Was a list of studies (included and excluded) } \\
\text { provided? } †\end{array}$} & Publication year & $0.86(0.81-0.92)$ & $<0.001$ \\
\hline & Non-pharmacological treatment§ & $0.42(0.20-0.86)$ & 0.017 \\
\hline $\begin{array}{l}\text { 6. Were the characteristics of the included studies } \\
\text { provided? }\end{array}$ & Publication year & $1.16(1.06-1.28)$ & 0.001 \\
\hline $\begin{array}{l}\text { 9. Were the methods used to combine the findings of } \\
\text { studies appropriate? }\end{array}$ & Corresponding author from Europe & $1.76(1.05-2.93)$ & 0.032 \\
\hline \multirow[t]{4}{*}{ 10. Was the likelihood of publication bias assessed? } & Publication year & $1.13(1.06-1.19)$ & $<0.001$ \\
\hline & Impact factor & $1.14(1.04-1.25)$ & 0.004 \\
\hline & Number of review authors & $1.12(1.01-1.24)$ & 0.034 \\
\hline & Non-pharmacological treatment§ & $1.62(1.01-2.59)$ & 0.047 \\
\hline
\end{tabular}

AMSTAR, Assessing the Methodological Quality of Systematic Reviews; CI, confidence interval; OR, odds ratio.

*The $p$ values of all HL tests were $>0.1$, indicating good model fit for all logistic regression analyses.

tAs an editorial policy of the Cochrane Library, all Cochrane reviews are required to provide protocol (item 1) and lists of included and excluded studies, and therefore all of them will fulfil requirements of AMSTAR Item 1 and 5. It appears to be methodologically unsound to included Cochrane reviews in regression analyses on these two items (we have attempted to do some and results are shown in online Appendix C). Hence, in this Table, regression analyses for Item 1 and 5 were only performed in non-Cochrane reviews.

‡Corresponding author from areas other than Europe were used as reference.

$\S$ Pharmacological treatment was used as the reference.

should be built to ensure availability of statistical expertise.

\section{Declaring conflict of interests explicitly}

It is well acknowledged that SRs funded commercially are less transparent, and are more likely to draw favourable conclusions to support the interventions (Lexchin et al. 2003; Jørgensen et al. 2006). Among included SRs, only $31.6 \%$ declared conflict of interests for both the included primary studies and the SR itself. While many journals now request that authors state conflict of interests for the SR, similar requirements do not always apply for the primary studies included in SRs. Lack of such information hinders assessment on possible conflict of interests stemming from primary studies. Adding such a requirement in the next version of PRISMA reporting guideline could further prevent this problem (Moher et al. 2009).

\section{Comparisons with similar studies}

Overall, quality of SRs on depression treatments is variable when compared with other clinical areas. A lower proportion of our sample reported PRISMA-like flow diagrams and treatment harms when compared to a sample of SRs from MEDLINE indexed in February
2014 (Page et al. 2016). However, when compared to SRs on hypertension treatments (Wu et al. 2016a), our sample performed better in the majority of AMSTAR items except for providing a protocol, conducting duplicate study selection and data extraction, and in considering risk of bias when drawing conclusions. Our sample also did better in stating sources of support than SRs on hypertension treatments ( $\mathrm{Wu}$ et al. 2016a), diabetes treatments (Wu et al. 2016b) and chronic obstructive pulmonary disease (Ho et al. 2015), while appeared inferior to SRs on Chinese herbal medicine (Chung et al. 2015).

\section{Strengths and limitations of this study}

This study has comprehensively assessed the methodological quality of SRs on depression treatments using the validated AMSTAR instrument (Shea et al. 2007, 2009). The results on each AMSTAR item were separated so as to inform future areas for improvement, as well as to alert evidence-users on potential bias associated with existing SRs. Meanwhile, our assessment result may be limited by varied reporting quality of the included SRs. For instance, 33.2\% of the included SRs did not describe whether duplicate study selection and data extraction were performed, and from the publication alone it is hard to ascertain whether such a procedure has been implemented 
(Wegewitz et al. 2016). Secondly, subgroup analysis and sensitivity analysis, which are important methodological aspects of SRs (Fleming et al. 2014), are not covered by the AMSTAR, thus, we did not assess those two domains in this study. The addition of these areas to AMSTAR has been proposed (Burda et al. 2016).

\section{Implications}

Despite a vast and growing body of summary literature relating to treatments for depression and despite a trend for improved reporting quality among SRs, many recently published SRs still do not meet minimum reporting criteria. Clinical decisions may not be made using the highest quality of evidence and subsequently, treatment decisions may be suboptimal for patients. Review authors should adhere to reporting standards for their work and use of these should be enforced by journal editors.

\section{Conclusion}

In conclusion, this study demonstrated that the methodological quality of SRs on depression treatments is low and variable. Areas that require urgent improvements include consideration of risk of bias among included studies when formulating conclusions, explicit declaration of conflict of interests, prevention of language and publication biases, using appropriate statistical techniques and ensuring timely updates of existing SRs. It is noteworthy that treatment harms are not reported in one third of the SRs on pharmacological treatments. Readers are encouraged to critically appraise quality of SRs before applying the results in decision-making. Peer reviewers and editors have a crucial role in promoting progress of SR quality by adhering to existing methodological and reporting standards promulgated by AMSTAR and PRISMA.

\section{Supplementary material}

The supplementary material for this article can be found at https://doi.org/10.1017/S2045796017000208.

\section{Acknowledgements}

The authors would like to acknowledge the significant contribution of other members of the research team who worked hard to identify, screen and select studies for inclusion in this work and who were also responsible for extracting data from the original manuscripts:
CHL Wong, JTM Chow, WKW Cheung, YF Yu, BHK Yip and RWS Sit.

\section{Financial Support}

This research received no specific grant from any funding agency, commercial or not-for-profit sectors.

\section{Conflicts of Interest}

None.

\section{Authorship statement}

All authors listed meet the authorship criteria according to the latest guidelines of the International Committee of Medical Journal Editors. VC and RH involved in study concept and design; YF in literature search. YF and RH helped in literature selection, data extraction and methodological quality assessments. XYW, SW and DT contributed in analysis and interpretation of data. VC and XYW drafted the manuscript. VC, DT, SW and XYW contributed to critical revision of the manuscript for important intellectual content. All authors are in agreement with the manuscript.

\section{Availability of Data and Materials}

Data set used for data analysis in this study is available from the corresponding author in request.

\section{References}

American Psychiatric Association (1980). Diagnostical and Statistical Manual of Mental Disorders (DSM-III). American Psychiatric Association: Washington, DC.

Burda BU, Holmer HK, Norris SL (2016). Limitations of a measurement tool to assess systematic reviews (AMSTAR) and suggestions for improvement. Systematic Reviews 5, 58.

Busse JW, Bruno P, Malik K, Connell G, Torrance D, Ngo T, Kirmayr K, Avrahami D, Riva JJ, Ebrahim S, Struijs PA, Brunarski D, Burnie SJ, LeBlanc F, Coomes EA, Steenstra IA, Slack T, Rodine R, Jim J, Montori VM, Guyatt GH (2014). An efficient strategy allowed English-speaking reviewers to identify foreign-language articles eligible for a systematic review. Journal of Clinical Epidemiology 67, 547-553.

Chung VCH, Ho RST, Wu XY, Fung DHY, Lai X, Wu JCW, Wong SYS (2015). Are meta-analyses of Chinese herbal medicine trials trustworthy and clinically applicable? A cross-sectional study. Journal of Ethnopharmacology 162, $47-54$. 
Cipriani A, Barbui C, Butler R, Hatcher S, Geddes J (2011). Depression in adults: drug and physical treatments. BMJ Clinical Evidence 2011, 1003.

Cochrane Community (beta) (2015). Database of Abstracts of Reviews of Effects (DARE).

Cohen JF, Korevaar DA, Wang J, Spijker R, Bossuyt PM (2015). Should we search Chinese biomedical databases when performing systematic reviews? Systematic Reviews 4, 23.

Dwan K, Altman DG, Arnaiz JA, Bloom J, Chan AW, Cronin E, Decullier E, Easterbrook PJ, Von Elm E, Gamble C, Ghersi D, Ioannidis JPA, Simes J, Williamson PR (2008). Systematic review of the empirical evidence of study publication bias and outcome reporting bias. PLoS ONE 3, e3081.

Egger M, Zellweger-Zähner T, Schneider M, Junker C, Lengeler C, Antes G (1997). Language bias in randomised controlled trials published in English and German. Lancet 350, 326-329.

Egger M, Smith GD, Sterne JA (2001). Uses and abuses of meta-analysis. Clinical Medicine 1, 478-484.

Fleming PS, Koletsi D, Seehra J, Pandis N (2014). Systematic reviews published in higher impact clinical journals were of higher quality. Journal of Clinical Epidemiology 67, 754-759.

Hamilton M (1960). A rating scale for depression. Journal of Neurology, Neurosurgery, and Psychiatry 23, 56-62.

Higgins J, Green S (2011). Cochrane Handbook for Systematic Reviews of Interventions Version 5.1.0 (updated March 2011). The Cochrane Collaboration. Available from www.cochrane-handbook.org.

Ho RS, Wu XY, Yuan JQ, Liu SY, Lai X, Wong SY, Chung VC (2015). Methodological quality of meta-analyses on treatments for chronic obstructive pulmonary disease: a cross-sectional study using the AMSTAR (Assessing the Methodological Quality of Systematic Reviews) tool. Npj Primary Care Respiratory Medicine 25, 14102.

Jørgensen AW, Hilden J, Gøtzsche PC (2006). Cochrane reviews compared with industry supported meta-analyses and other meta-analyses of the same drugs: systematic review. BMJ 333, 782.

Jüni P, Holenstein F, Sterne J, Bartlett C, Egger M (2002). Direction and impact of language bias in meta-analyses of controlled trials: empirical study. International Journal of Epidemiology 31, 115-123.

Lexchin J, Bero LA, Djulbegovic B, Clark O (2003). Pharmaceutical industry sponsorship and research outcome and quality: systematic review. BMJ 326, 1167-1170.

Moher D, Tsertsvadze A (2006). Systematic reviews: when is an update an update? Lancet 367, 881-883.

Moher D, Tetzlaff J, Tricco AC, Sampson M, Altman DG (2007). Epidemiology and reporting characteristics of systematic reviews. PLoS Medicine 4, e78.

Moher D, Liberati A, Tetzlaff J, Altman DG (2009). Preferred reporting items for systematic reviews and meta-analyses: the PRISMA statement. BMJ 339, b2535.

Moja LP, Telaro E, D'Amico R, Moschetti I, Coe L, Liberati A (2005). Assessment of methodological quality of primary studies by systematic reviews: results of the metaquality cross sectional study. BMJ 330, 1053.

Morrison A, Polisena J, Husereau D, Moulton K, Clark M, Fiander M, Mierzwinski-Urban M, Clifford T, Hutton B,
Rabb D (2012). The effect of English-language restriction on systematic review-based meta-analyses: a systematic review of empirical studies. International Journal of Technology Assessment in Health Care 28, 138-144.

Mulrow CD, Cook DJ, Davidoff F (1997). Systematic reviews: critical links in the great chain of evidence. Annals of Internal Medicine 126, 389-391.

Mykletun A, Bjerkeset O, Overland S, Prince M, Dewey M, Stewart R (2009). Levels of anxiety and depression as predictors of mortality: the HUNT study. British Journal of Psychiatry 195, 118-125.

Page MJ, Shamseer L, Altman DG, Tetzlaff J, Sampson M, Tricco AC, Catalá-López F, Li L, Reid EK, Sarkis-Onofre R, Moher D (2016). Epidemiology and reporting characteristics of systematic reviews of biomedical research: a cross-sectional study. PLoS Medicine 13, e1002028.

Papageorgiou SN, Papadopoulos MA, Athanasiou AE (2011). Evaluation of methodology and quality characteristics of systematic reviews in orthodontics. Orthodontics and Craniofacial Research 14, 116-137.

Remschmidt C, Wichmann O, Harder T (2014). Methodological quality of systematic reviews on influenza vaccination. Vaccine 32, 1678-1684.

Sequeira-Byron P, Fedorowicz Z, Jagannath VA, Sharif MO (2011). An AMSTAR assessment of the methodological quality of systematic reviews of oral healthcare interventions published in the Journal of Applied Oral Science (JAOS). Journal of Applied Oral Science 19, 440-447.

Shea B, Grimshaw J, Wells G, Boers M, Andersson N, Hamel C, Porter A, Tugwell P, Moher D, Bouter L (2007). Development of AMSTAR: a measurement tool to assess the methodological quality of systematic reviews. BMC Medical Research Methodology 7, 10.

Shea B, Hamel C, Wells GA, Bouter L, Kristjansson E, Grimshaw J, Henry D, Boers M (2009). AMSTAR is a reliable and valid measurement tool to assess the methodological quality of systematic reviews. Journal of Clinical Epidemiology 62, 1013-1020.

Wegewitz U, Weikert B, Fishta A, Jacobs A, Pieper D (2016). Resuming the discussion of AMSTAR: what can (should) be made better? BMC Medical Research Methodology 16, 111.

World Health Organization (2012). Depression. http://www. who.int/mediacentre/factsheets/fs369/en/

Wu XY, Tang JL, Mao C, Yuan JQ, Qin Y, Chung VCH (2013). Systematic reviews and meta-analyses of traditional Chinese medicine must search Chinese databases to reduce language bias. Evidence-based Complementary and Alternative Medicine: eCAM 2013, 812179.

Wu XY, Du XJ, Ho RS, Lee CC, Yip BH, Wong MC, Wong SY, Chung VC (2016a). Characteristics and methodological quality of meta-analyses on hypertension treatments-a cross-sectional study. Journal of Clinical Hypertension 19, 137-142.

Wu XY, Lam VC, Yu YF, Ho RS, Feng Y, Wong CH, Yip BH, Tsoi K, Wong SY, Chung VC (2016b). Epidemiological characteristics and methodological quality of meta-analyses on diabetes mellitus treatment: a systematic review. European Journal of Endocrinology 175, 353-360. 